\title{
A simple model predicts how warming simplifies wild food webs
}

Eoin J. O’Gorman ${ }^{1, *}$, Owen L. Petchey ${ }^{2}$, Katy J. Faulkner ${ }^{3}$, Bruno Gallo ${ }^{4}$,

Timothy A.C. Gordon ${ }^{5}$, Joana Neto-Cerejeira², Jón S. Ólafsson ${ }^{6}$, Doris E.

Pichler $^{4}$, Murray S.A. Thompson ${ }^{7}$, and Guy Woodward ${ }^{4, *}$.

${ }^{1}$ School of Biological Sciences, University of Essex, Wivenhoe Park, Colchester, CO4 3SQ, UK.

${ }^{2}$ Institute of Evolutionary Biology and Environmental Studies, University of Zurich,

Winterthurerstrasse 190, CH-8057 Zurich, Switzerland.

${ }^{3}$ School of Life Sciences, Gibbet Hill Campus, University of Warwick, Coventry, CV4 7AL, UK.

${ }^{4}$ Department of Life Sciences, Imperial College London, Silwood Park Campus, Buckhurst Road, Ascot, Berkshire, SL5 7PY, UK.

${ }^{5}$ Biosciences, College of Life and Environmental Sciences, University of Exeter, Stocker Road, Exeter, EX4 4QD, UK.

${ }^{6}$ Marine and Freshwater Research Institute, Skúlagata 4, 101 Reykjavík, Iceland.

${ }^{7}$ Centre for Environment, Fisheries \& Aquaculture Science, Pakefield Road, Lowestoft, Suffolk, NR33 OHT, UK.

Running head: Warming simplifies food webs

Type of paper: Letter

* Corresponding authors: Eoin O'Gorman; Guy Woodward

E-mail: e.ogorman@essex.ac.uk; guy.woodward@imperial.ac.uk

Tel: +44 $1206876389 ;+442075942237$

Keywords: global warming, natural experiment, Arctic, aquatic, ecological networks, allometric diet breadth model 
1 Warming increases the metabolic demand of consumers ${ }^{1}$, strengthening their feeding

2 interactions ${ }^{2}$. This could alter energy fluxes ${ }^{3-5}$ and even amplify extinction rates within

3 the food web ${ }^{6-8}$. Such effects could simplify the structure and dynamics of ecological 4 networks ${ }^{9,10}$, although an empirical test in natural systems has been lacking. Here, we

5 tested this hypothesis by characterising $\sim \mathbf{5 0 , 0 0 0}$ directly observed feeding interactions

6 across 14 naturally heated stream ecosystems ${ }^{11-15}$. We found that higher temperature

7 simplified food web structure and shortened the pathways of energy flux between consumers and resources. A surprisingly simple allometric diet breadth model ${ }^{10,16}$ predicted $68-82 \%$ of feeding interactions and the effects of warming on key food web properties. We used model simulations to identify the underlying mechanism as a change in the relative diversity and abundance of consumers and their resources. This shows how warming can reduce the stability of aquatic ecosystems by eroding the structural integrity of the food web. Given these fundamental drivers, such responses are expected to be manifested more broadly and could be predicted using our modelling framework and knowledge of how warming alters some routinely measured characteristics of organisms.

All natural systems contain complex food webs, whose stability is shaped by nonrandom structural properties ${ }^{17}$, e.g. the strength of consumer-resource interactions ${ }^{18,19}$ and the flow of energy from many abundant small species into progressively fewer large species, especially in the aquatic realm ${ }^{20}$. Global warming could disrupt these patterns, yet we lack high quality field data to test and validate predictive models of temperature effects on food webs. In theory, consumers should exert stronger feeding pressure on the biomass stocks of lower trophic levels in warmer environments ${ }^{3,4}$, but may struggle to meet their rising energy demands ${ }^{7,8}$. This could lead to shorter food chains ${ }^{6}$, simpler food webs ${ }^{9}$, less efficient energy flux ${ }^{5}$, and an altered distribution of biomass through the food $\mathrm{web}^{21}$. 
To test these expectations, we exhaustively characterised food web interactions for 14

27 geothermally heated streams in Iceland using dietary analysis (see Methods). The streams occur within $1.5 \mathrm{~km}$ of each other in a pristine mountain landscape (Fig. S1), free from anthropogenic influences apart from occasional sheep grazing. The streams are very similar in their physical and chemical properties and yet vary in temperature from $5-25{ }^{\circ} \mathrm{C}$ due to indirect heating of groundwater through the bedrock (Tables S1-S3). Since the streams occur in the same catchment, they avoid the biogeographical differences associated with other natural gradients in temperature (e.g. latitude or altitude) $)^{22}$. This study system thus acts as a space-for-time proxy, where temperature effects on food web structure can be investigated in a wild setting with all the complexity and realism of natural ecosystems ${ }^{22}$.

We used an allometric diet breadth model $(\mathrm{ADBM})^{10,16}$, parameterised with data on the average body mass and population abundance of species sampled in each stream in August 2008 (i.e. no a priori information on feeding links), to predict the structure of each food web (see Methods). We then examined how several properties of the ADBM-predicted food webs varied with stream temperature, finding significant linear relationships for four key metrics related to food chain length, complexity, energy flux, and biomass distribution (Fig. S2). This allowed us to formulate four hypotheses (H1-4) that could be tested with an empirical quantification of feeding links in the system. We anticipate that, as stream temperature increases, there will be: (H1) a reduction in mean trophic level; (H2) a decrease in connectance; (H3) shorter pathways of energy flux through the food web; and (H4) an increasing biomass of consumers relative to their resources.

We tested our predictions by characterising the actual food web structure of each stream based on almost 50,000 gut content observations (see Methods). There was a simplification of food web structure as stream temperature increased, from a diffuse, reticulate network (Fig. 1a) to one with fewer and shorter chains (Fig. 1b). In support of H1, 
mean trophic level was lower in the warmer streams (Fig. 1c), with herbivorous interactions becoming increasingly dominant. This appeared to be driven by a disproportionate loss of consumer species, relative to resources, as stream temperature increased (Fig. S3). Consumer losses likely occurred as they were unable to meet the greater metabolic demands of the warmer environment ${ }^{1,7}$ and/or withstand increased predation by an apex predator, brown trout, which cannot persist in the coldest streams due to its own metabolic constraints ${ }^{13,14}$. Warmer food webs were also less connected (Fig. 1d), as expected in H2, suggesting they will be more sensitive to secondary extinctions $s^{23,24}$ and dominated by more specialised consumers, with energy channelled through fewer and stronger links ${ }^{9}$. Similar patterns were obtained when the same streams were sampled again in April 2009 (Fig. S4a,b).

To assess how these structural changes altered energy flux through the food web, we calculated the lengths and angles of all pairwise consumer-resource links in $\log _{10}$ (body mass) and $\log _{10}$ (abundance) space $^{25}$ (see Fig. 2a,b for definitions of these terms). The average pathway of energy flux through the food web was shorter in warmer streams (Fig. 2c,d), due to a reduction in mean link length as temperature increased (Fig. 2e). This supports H3 and points to stronger feeding pressure in the warmer streams ${ }^{13,14}$, with the abundance of resources suppressed relative to their consumers (Fig. S5b). A link angle of $-45^{\circ}$ means that resource biomass equals consumer biomass ${ }^{25}$ (Fig. 2a) and mean link angle became progressively smaller than this at higher temperatures (Fig. 2f). This indicates that the biomass of consumers was on average greater than the biomass of their resources in the warmer streams (Fig. S5c, S6), as predicted in H4. Inverted biomass pyramids are promoted by stronger top-down control, generalist feeding, larger predators, and higher trophic transfer efficiency ${ }^{21,26}$, all of which have been documented to increase with stream temperature in the Hengill system ${ }^{12-14}$. They can only persist, however, if resources are replenished rapidly enough to meet the metabolic demands of consumers ${ }^{12}$, i.e. the standing stock of resources is 
low, but production is high enough to maintain consumer biomass through time. Such topheaviness is increasingly documented in nature when consumer pressure or anthropogenic disturbance is especially powerful (e.g. in marine fisheries), but these systems are less stable than their pyramidal counterparts ${ }^{21,27}$. We found similar patterns for mean link angle, but no effect on mean link length from the April 2009 sampling (Fig. S4c,d), suggesting that effects of temperature on the latter in August 2008 should be treated with caution.

Our model accurately predicted a higher proportion of empirically observed feeding interactions than previously documented for high quality food webs ${ }^{16}: 75 \pm 3.9 \%$ (mean \pm standard deviation) across all 14 streams (Fig. S7). This shows that the ADBM can be a useful tool for predicting ecological networks ${ }^{16}$, at least for size-structured aquatic ecosystems like our study streams ${ }^{12}$, even when interaction data are limited, as is the case for most studies to date ${ }^{28}$. Our empirical measures of food web structure and energy flux were also strongly correlated with the ADBM predictions, although deviation of the slope from the 1:1 line suggests the model did not produce an accurate quantitative prediction of connectance (Fig. S8). Our results indicate that the ADBM can also predict the impacts of temperature on natural food webs, using simple information that is routinely collected in ecological field studies. Further testing of the model with other highly resolved food web datasets from experiments that have manipulated warming in a controlled fashion would validate this suggestion more broadly.

As a final exploratory step, we investigated the underpinning mechanisms by using the

96 ADBM to simulate food webs after changing one of the three major input variables: species 97 identity, average body mass, and population abundance. By randomly choosing species from the regional species pool ('sp' scenario), we disrupted the trophic structure of any given stream and thus the relationship between stream temperature and the ratio of consumer to resource species richness (Fig. 3a). By randomly choosing a mean body mass (' $M$ ' scenario) 
101 or population abundance (' $N$ ' scenario) for each species from the same trophic groups in the 102 regional dataset, we disrupted the relationship between stream temperature and the ratio of 103 consumer to resource body mass or abundance, respectively (Fig. 3b,c). For each scenario, 104 we then simulated 1,000 food webs for each of our 14 study streams after randomising one 105 input variable and fixing the values of the other two variables as close to the real stream as 106 possible (see Methods).

107 Our 'sp' scenario removed the effect of temperature on mean trophic level and connectance (Fig. 3d,e), with negligible effects of the other two scenarios. This suggests that

109 the relative biodiversity of consumers and resources is a key determinant of these food web 110 properties. We used the 14,000 food webs simulated under the 'sp' scenario to explore this 111 effect, independent of temperature, and found that both mean trophic level and connectance 112 increase with the ratio of consumer to resource species richness (Fig. 4a,b). Thus, the 113 disproportionate loss of consumer species, which is widely predicted in response to 114 warming $^{6-8}$, should lead to reductions in these food web properties.

115 While all three randomisation scenarios disrupted temperature effects on link lengths 116 and angles, our ' $N$ ' scenario had by far the greatest effect (Fig. 3f,g), suggesting the ratio of 117 consumer to resource abundance is the principal determinant of energy flux. We used the 118 food webs simulated under the ' $N$ ' scenario to explore this effect, independent of temperature, 119 and found that link lengths and angles become smaller as consumers approach the abundance 120 of their resources (Fig. 4c,d). Thus, stronger top-down control that alters the shape of trophic 121 abundance pyramids, which is often reported in warmer environments ${ }^{3,4}$, will suppress energy 122 flux through the food web.

123 Our study is one of the first to show systematic impacts of temperature on wild food 124 webs (e.g. see also $\left.{ }^{29}\right)$. Most riverine ecosystems in Europe and North America fall within the 125 studied temperature gradient of $5-25^{\circ} \mathrm{C}^{30}$ and so our results should be indicative of changes 
126 in food web structure due to future warming within this range. Our findings highlight the

127 importance of monitoring species interactions for successful management of ecosystems ${ }^{31}$,

128 given that trophic structure is so sensitive to environmental change. For example, mean

129 trophic level is increasingly used in fisheries management to identify overfishing at the top of

130 the food $w^{32}{ }^{32}$, while connectance is a useful indicator of resistance to invasion ${ }^{33}$ and

131 robustness against biodiversity $\operatorname{loss}^{23,24}$. We identified changes in the relative biodiversity or

132 abundance of consumers and resources at higher temperatures as key mechanisms driving the

133 observed effects. Such changes are also elicited by anthropogenic activities like

134 overexploitation and habitat degradation ${ }^{32,34}$, emphasising how the structure and stability of

135 ecological networks may be threatened by a host of stressors. The predictive power of our

136 model shows how the impact of these stressors could be anticipated and ultimately mitigated

137 more broadly. These findings now need to be tested in a range of food webs from marine,

138 freshwater, and terrestrial realms to gauge their potential universality.

\section{Acknowledgements}

140 We thank Gísli Már Gíslason for providing research support and laboratory facilities.

141 We thank Rebecca L. Kordas (Imperial College London, UK), Georgina Adams (University

142 College London, UK), Eileen J. Cox and Luis Moliner Cachazo (Natural History Museum,

143 London, UK), Iris Hansen and Sigurdur Oskar Helgason (Marine and Freshwater Research

144 Institute, Iceland), and Paula C. Furey (St Catherine University, USA) for help with

145 taxonomic identification. We acknowledge funding from NERC (NE/I009280/2,

146 NE/F013124/1, NE/L011840/1, NE/M020843/1), Imperial College London's Masters in

147 Ecology, Evolution \& Conservation, and the University of Zurich Research Priority

148 Programme Global Change and Biodiversity. 
EJOG, OLP, and GW were responsible for funding application, research design, and

151 planning. EJOG, KJF, BG, TACG, JNC, JSÓ, DEP, and MSAT collected the data. EOG and

152 OLP analysed the data. All authors wrote the paper.

\section{References}

1541 Brown, J. H., Gillooly, J. F., Allen, A. P., Savage, V. M. \& West, G. B. Toward a 155 metabolic theory of ecology. Ecology 85, 1771-1789 (2004).

1562 Rall, B. C. et al. Universal temperature and body-mass scaling of feeding rates. 157 Philosophical Transactions of the Royal Society B: Biological Sciences 367, 2923$2934(2012)$.

1593 O’Connor, M. I., Piehler, M. F., Leech, D. M., Anton, A. \& Bruno, J. F. Warming and 160 resource availability shift food web structure and metabolism. PLoS Biology 7, $161 \quad$ e1000178 (2009).

1624 Shurin, J. B., Clasen, J. L., Greig, H. S., Kratina, P. \& Thompson, P. L. Warming 163 shifts top-down and bottom-up control of pond food web structure and function. 164 Philosophical Transactions of the Royal Society B: Biological Sciences 367, 3008$165 \quad 3017(2012)$.

1665 Schwarz, B. et al. Warming alters energetic structure and function but not resilience 167 of soil food webs. Nature Climate Change 7, 895-900 (2017).

1686 Petchey, O. L., McPhearson, P. T., Casey, T. M. \& Morin, P. J. Environmental 169 warming alters food-web structure and ecosystem function. Nature 402, 69-72 (1999).

1707 Vucic-Pestic, O., Ehnes, R. B., Rall, B. C. \& Brose, U. Warming up the system: 171 higher predator feeding rates but lower energetic efficiencies. Global Change Biology 172 17, 1301-1310 (2011). 
1738 Fussmann, K. E., Schwarzmüller, F., Brose, U., Jousset, A. \& Rall, B. C. Ecological 174 stability in response to warming. Nature Climate Change 4, 206-210 (2014).

1759 Binzer, A., Guill, C., Rall, B. C. \& Brose, U. Interactive effects of warming, 176 eutrophication and size-structure: impacts on biodiversity and food-web structure. 177 Global Change Biology 22, 220-227 (2016).

17810 Petchey, O. L., Brose, U. \& Rall, B. C. Predicting the effects of temperature on food 179 web connectance. Philosophical Transactions of the Royal Society B: Biological $180 \quad$ Sciences 365, 2081-2091 (2010).

$18111 \quad$ Friberg, N. et al. Relationships between structure and function in streams contrasting 182 in temperature. Freshwater Biology 54, 2051-2068 (2009).

18312 O'Gorman, E. J. et al. Unexpected changes in community size structure in a natural 184 warming experiment. Nature Climate Change 7, 659-666 (2017).

18513 O'Gorman, E. J. et al. Impacts of warming on the structure and functioning of aquatic 186 communities: individual- to ecosystem-level responses. Advances in Ecological $187 \quad$ Research 47, 81-176 (2012).

18814 O'Gorman, E. J. et al. Temperature effects on fish production across a natural thermal 189 gradient. Global Change Biology 22, 3206-3220 (2016).

19015 Adams, G. et al. Diatoms can be an important exception to temperature-size rules at 191 species and community levels of organization. Global Change Biology 19, 3540-3552 192 (2013).

19316 Petchey, O. L., Beckerman, A. P., Riede, J. O. \& Warren, P. H. Size, foraging, and 194 food web structure. Proceedings of the National Academy of Sciences of the United $195 \quad$ States of America 105, 4191-4196 (2008).

19617 Pimm, S. L. Food Webs. (University of Chicago Press, 1982).

19718 Allesina, S. \& Tang, S. Stability criteria for complex ecosystems. Nature 483, 205- 
19919 McCann, K., Hastings, A. \& Huxel, G. R. Weak trophic interactions and the balance of nature. Nature 395, 794-798 (1998).

$20120 \quad$ Elton, C. S. Animal Ecology. (Sidgwick \& Jackson, 1927).

20221 McCauley, D. J. et al. On the prevalence and dynamics of inverted trophic pyramids and otherwise top-heavy communities. Ecology Letters 21, 439-454 (2018).

20422 O'Gorman, E. J. et al. Climate change and geothermal ecosystems: natural laboratories, sentinel systems, and future refugia. Global Change Biology 20, 32913299 (2014).

23 Dunne, J. A., Williams, R. J. \& Martinez, N. D. Network structure and biodiversity loss in food webs: robustness increases with connectance. Ecology Letters 5, 558-567 (2002).

210

24 Gilbert, A. J. Connectance indicates the robustness of food webs when subjected to species loss. Ecological Indicators 9, 72-80 (2009).

21225 Cohen, J. E., Schittler, D. N., Raffaelli, D. G. \& Reuman, D. C. Food webs are more 213 than the sum of their tritrophic parts. Proceedings of the National Academy of Sciences 106, 22335-22340 (2009).

21526 Woodson, C. B., Schramski, J. R. \& Joye, S. B. A unifying theory for top-heavy ecosystem structure in the ocean. Nature communications 9, 23 (2018).

21727 Neutel, A. M., Heesterbeek, J. A. P. \& de Ruiter, P. C. Stability in real food webs: $218 \quad$ Weak links in long loops. Science 296, 1120-1123 (2002).

21928 Ings, T. C. et al. Ecological networks - beyond food webs. Journal of Animal Ecology 78, 253-269 (2009).

22129 Tunney, T. D., McCann, K. S., Lester, N. P. \& Shuter, B. J. Effects of differential habitat warming on complex communities. Proceedings of the National Academy of 
Sciences 111, 8077-8082 (2014).

22430 Van Vliet, M., Ludwig, F., Zwolsman, J., Weedon, G. \& Kabat, P. Global river 225 temperatures and sensitivity to atmospheric warming and changes in river flow. Water $226 \quad$ Resources Research 47 (2011).

22731 Gray, C. et al. Ecological networks: the missing links in biomonitoring science. $228 \quad$ Journal of Applied Ecology 51, 1444-1449 (2014).

22932 Branch, T. A. et al. The trophic fingerprint of marine fisheries. Nature 468, 431-435 $230 \quad(2010)$.

23133 Smith-Ramesh, L. M., Moore, A. C. \& Schmitz, O. J. Global synthesis suggests that 232 food web connectance correlates to invasion resistance. Global Change Biology 23, $233 \quad 465-473(2017)$.

23434 Barnes, A. D. et al. Consequences of tropical land use for multitrophic biodiversity 235 and ecosystem functioning. Nature Communications 5, 5351 (2014).

236 
Fig. 1. Temperature effects on food web properties. Food webs for the (a) coldest and (b) warmest stream in the system, where circles are species, grey lines are feeding interactions, and the size of the circles is proportional to the population biomass of each species in the stream. Note the reduction in the number of consumer species in the food web for the warm stream and the 'thinning out' of feeding interactions compared to the cold stream. There was a reduction in (c) mean trophic level $\left(y=1.536-0.0054 x, F_{1,12}=16.10, p<0.001, r^{2}=0.54\right)$ and $(\mathbf{d})$ directed connectance $\left(y=0.220-0.0023 x, F_{1,12}=18.93, p<0.001, r^{2}=0.58\right)$ as stream temperature increased (see Methods for definitions of these food web properties).

Fig. 2. Temperature effects on energy flux. a, The length of a trophic link (grey line) is defined as the sum of the number of orders of magnitude of difference in body mass (L1) and abundance (L2) between a consumer $(\mathrm{C})$ and a resource $(\mathrm{R})^{25}$. The angle (A) of a trophic link measures the rate of change in biomass from a consumer to a resource ${ }^{25}$. Here, consumer biomass (mass $\times$ abundance $\left.=10^{0} \times 10^{0}=1 \mathrm{mg} \mathrm{m}^{-2}\right)$ equals resource biomass $\left(10^{-8} \times 10^{8}=1\right.$ $\mathrm{mg} \mathrm{\textrm {m } ^ { - 2 }}$ ), resulting in a link angle of $-45^{\circ} . \mathbf{b}$, A decline in resource abundance and an increase in consumer abundance (relative to panel a) results in a shorter link length and a less negative link angle. Here, consumer biomass $\left(10^{0} \times 10^{2}=100 \mathrm{mg} \mathrm{m}^{-2}\right)$ is greater than resource

254 biomass $\left(10^{-8} \times 10^{6}=0.01 \mathrm{mg} \mathrm{m}^{-2}\right)$, resulting in a link angle of $-27^{\circ}$. Trivariate food webs for the (c) coldest and (d) warmest stream in the system, where circles are species, grey lines are feeding interactions, and the thick black lines represent the mean link length and mean link angle of the food web. There was (e) a reduction in mean link length $(y=9.067-0.0335 x$, $\left.F_{1,12}=5.04, p<0.001, r^{2}=0.24\right)$ and (f) a smaller (i.e. less negative) mean link angle $(y=$ $\left.-52.30+0.797 x, F_{1,12}=37.28, p<0.001, r^{2}=0.74\right)$ as stream temperature increased. 

mean ( \pm standard deviation) of linear regression slopes between food web properties and stream temperature for 1,000 randomisations are shown in the plots. The black dashed line represents a regression slope of zero between a food web property and temperature, i.e. the property is independent of temperature. The solid and dashed grey lines represent the empirical and ADBM-predicted regression slope of each food web property against temperature, respectively. a, The 'sp' scenario randomises the species found in a stream and thus the ratio of consumer to resource species richness. $\mathbf{b}$, The ' $M$ ' scenario randomises the average body mass of species in the stream and thus the ratio of consumer to resource body mass. c, The ' $N$ ' scenario randomises the population abundance of species in the stream and thus the ratio of consumer to resource abundance. The effect of temperature on (d) mean trophic level and (e) connectance is removed by the 'sp' scenario. The effect of temperature on (f) mean link length and (g) mean link angle is removed by the ' $N$ ' scenario. In all other cases, even if the randomisation scenario disrupts the empirical and ADBM-predicted patterns, it maintains the directionality of the temperature effect on the food web property.

Fig. 4. Key determinants of food web properties. Effect of the ratio of consumer to resource species richness (independent of temperature) on (a) mean trophic level $(y=0.847+$ $\left.1.4044 x, r^{2}=0.94\right)$ and $(\mathbf{b})$ connectance $\left(y=0.227+0.1952 x, r^{2}=0.35\right)$ in 1,000 food webs simulated for each of the 14 streams under the 'sp' scenario (cf. Fig. 3). Effect of the log ratio of consumer to resource abundance (independent of temperature) on (c) mean link length $(y=$ $\left.6.284-0.5105 x, r^{2}=0.32\right)$ and (d) mean link angle $\left(y=-20.37+3.297 x, r^{2}=0.13\right)$ in 1,000

281 food webs simulated for each of the 14 streams under the ' $N$ ' randomisation scenario (cf. Fig.

282 3). Parameter estimates are the mean intercept, slope, and $r^{2}$ values from 1,000 linear regressions of the relationship across streams (i.e. one regression for each randomisation). 
Fig. 1

a

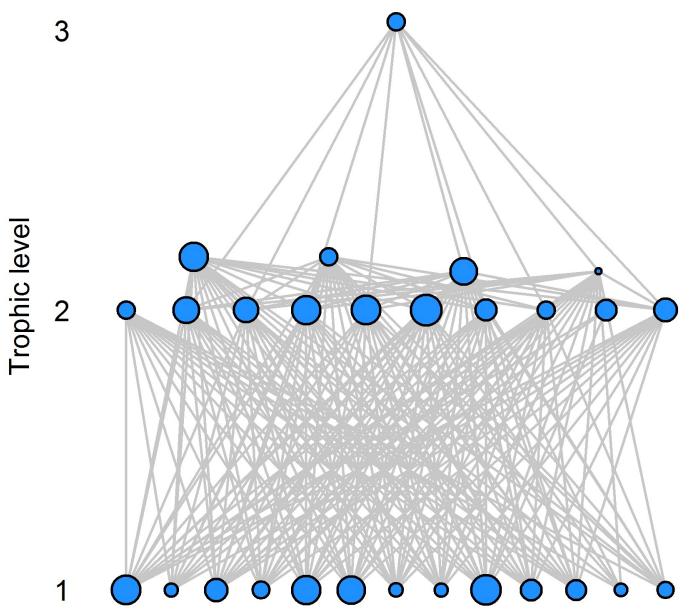

c



Coldest study stream b Warmest study stream

d

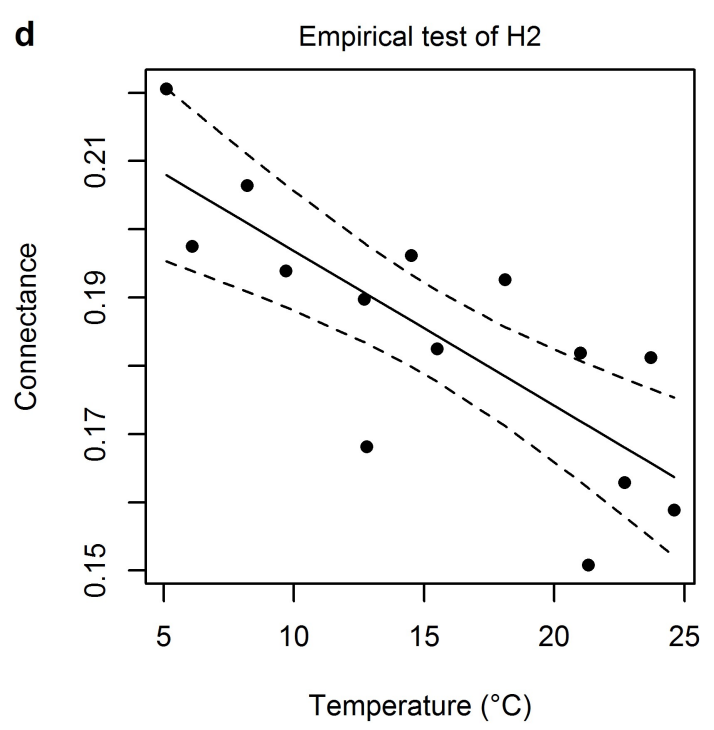

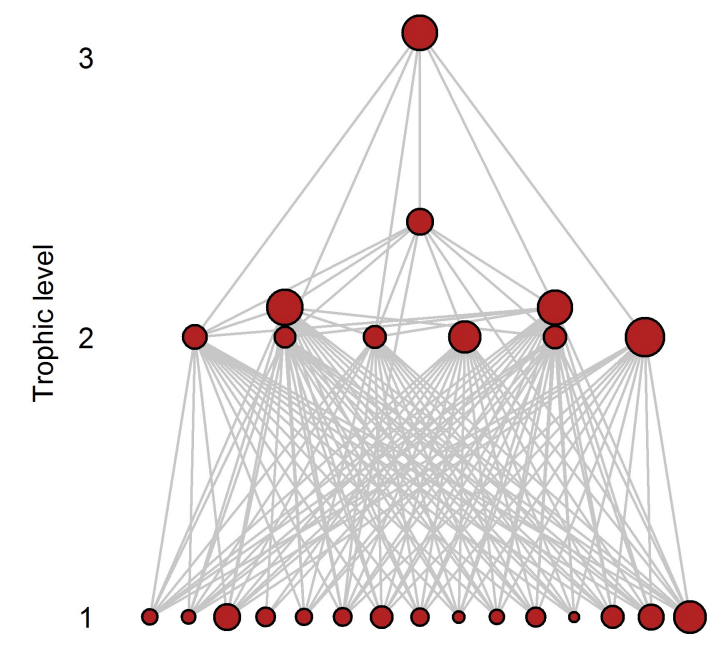


Fig. 2
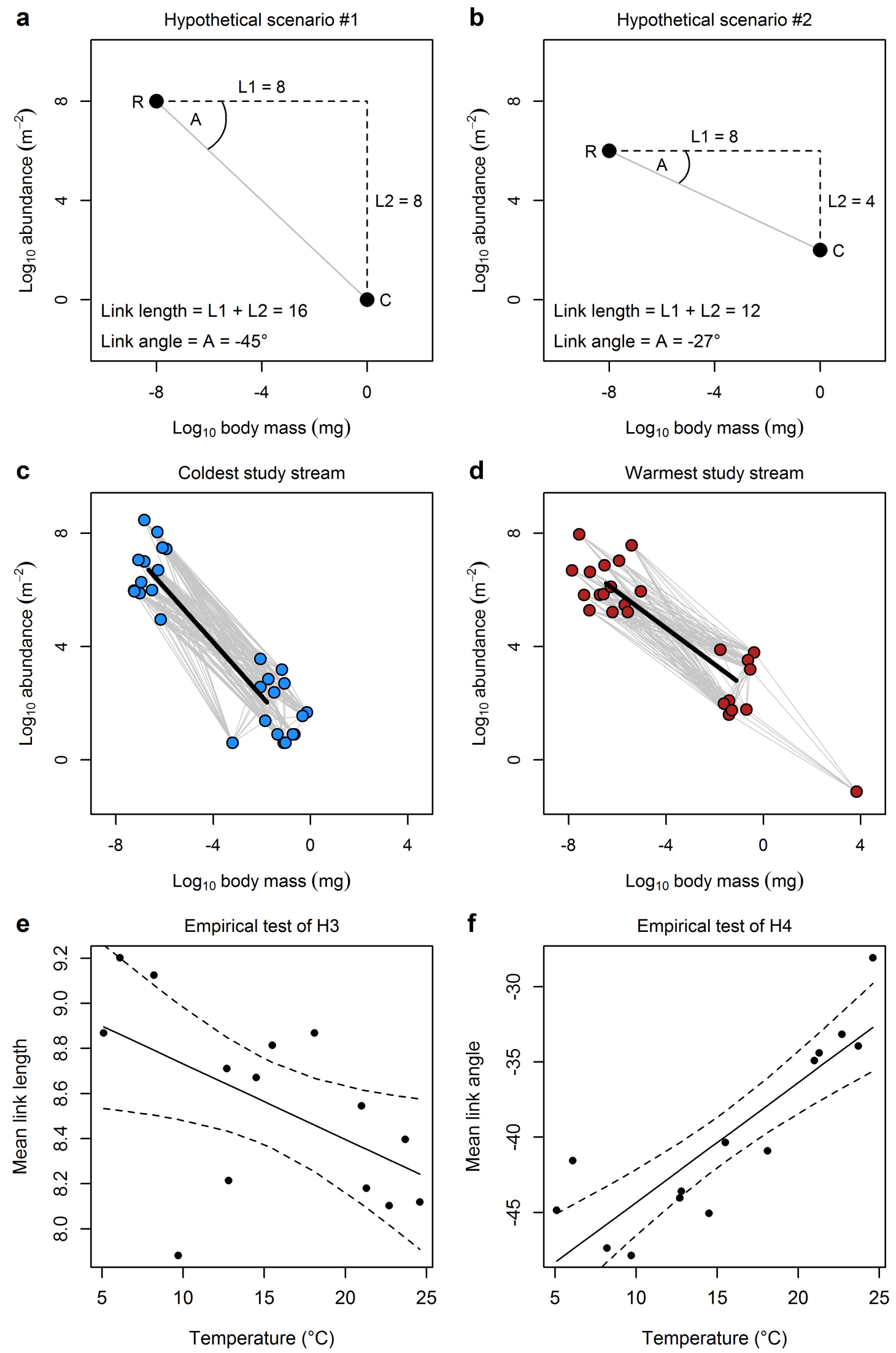
289 Fig. 3
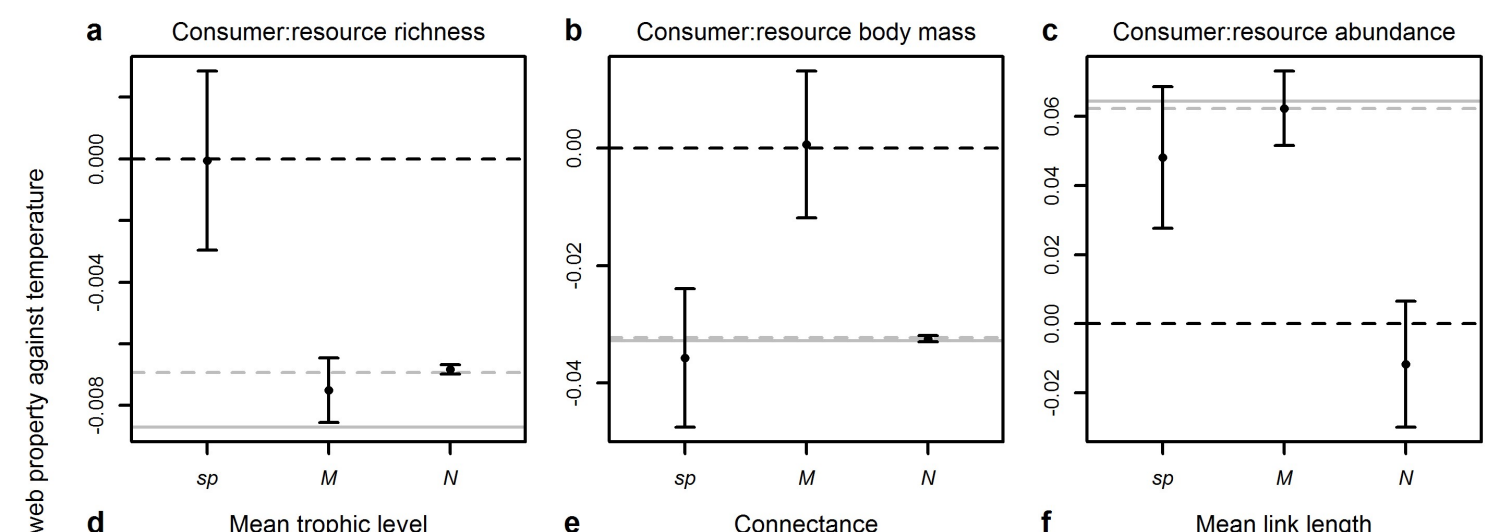

Top row:

Tests of randomisation success

Bottom row:

Effects of randomisations on properties

of the food webs

$X$-axis labels:

'sp' randomises trophic structure

' $\mathrm{N}$ ' randomises abundance structure

- - Independent of temperature

ADBM-predicted temperature effect

- Empirical tempe
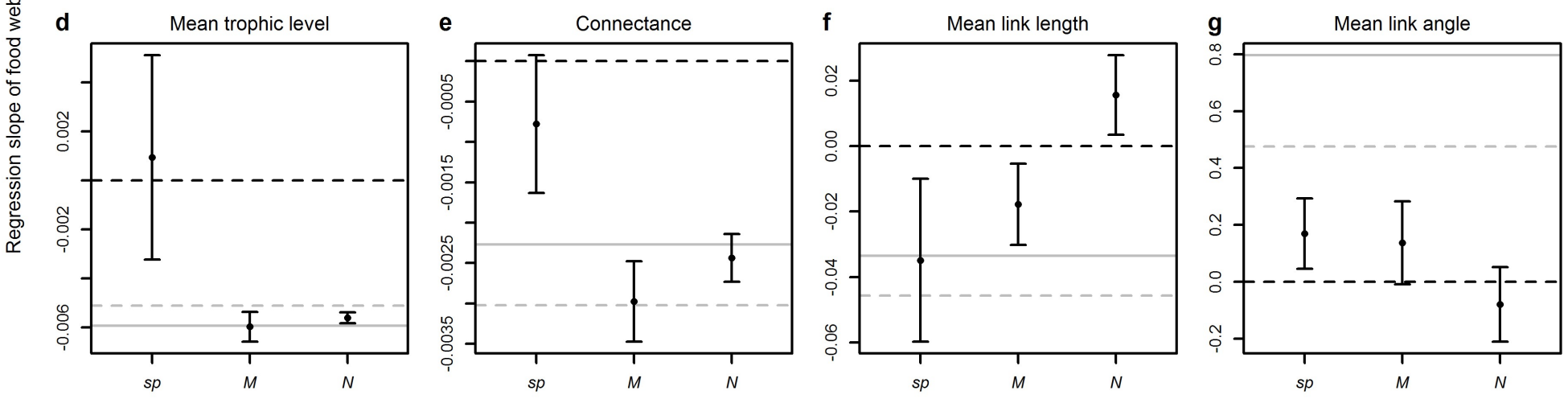

290

Randomisation scenario 
Fig. 4

a

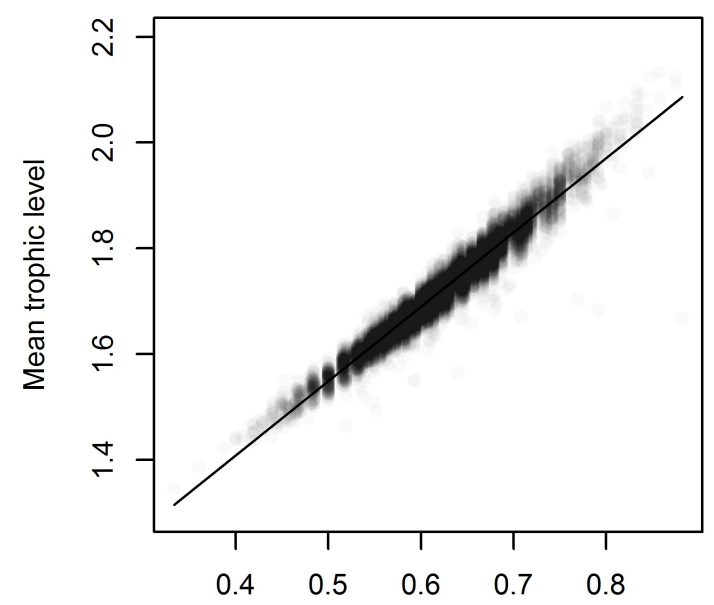

b



Ratio of consumer to resource species richness

C

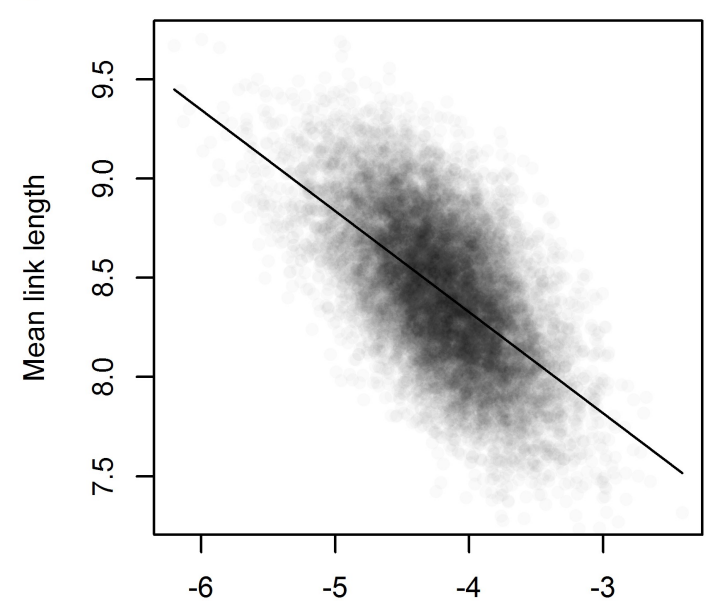

d

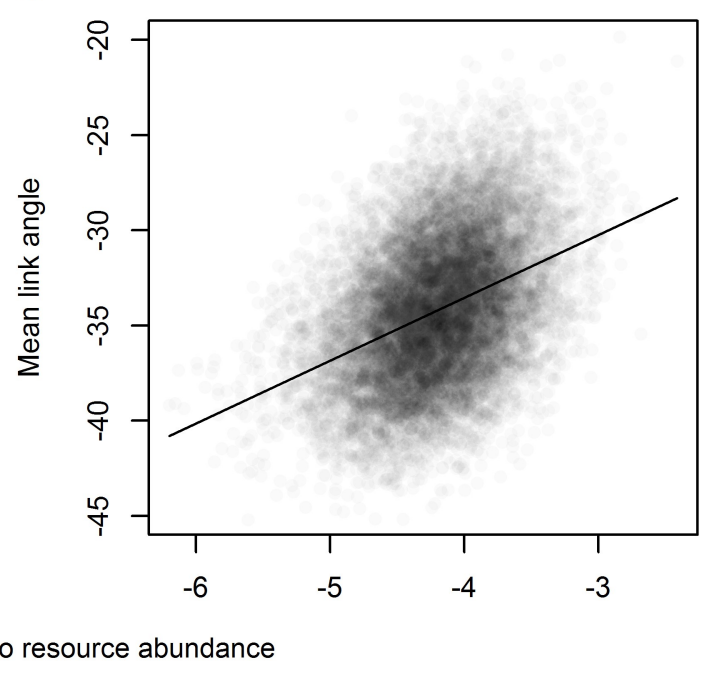


Methods

Stream sampling

Streams were sampled in August 2008 and April 2009 to quantify the three major

297 trophic groups in the system: benthic diatoms (three stone scrapes per stream),

298 macroinvertebrates (five Surber samples per stream), and fish (three-run depletion

299 electrofishing). Yield-effort curves were constructed to verify the efficiency of sampling ${ }^{13}$.

300 We focus on the August 2008 data throughout because they represent the height of the

301 growing season, whereas the April 2009 data are from a time of the year when the streams are

302 in transition. Thus, we only use the latter to determine how consistent the observed patterns

303 are through time. Diatoms and macroinvertebrates were identified to species level under the

304 microscope and counted to estimate population abundance, which was scaled to number of

305 individuals per $\mathrm{m}^{2}$ based on sampling areas. Average body mass (in milligrams of dry

306 weight) was estimated from linear measurements for at least ten individuals of every species

307 and published length-weight relationships (Tables S4 and S5). Note that diatoms could only

308 be reliably identified to genus level in gut contents, so we calculated the total abundance and

309 abundance-weighted mean body mass of each diatom genus from the species-level data.

310 Nevertheless, we refer to all taxa as species throughout this paper. Body mass measurements

311 of the only fish in the system (brown trout, Salmo trutta), were taken on a portable mass

312 balance and converted to dry weight according to a wet weight to dry weight relationship ${ }^{12}$.

313 Precise details of the study system and stream sampling are given in Supplementary Methods.

\section{Overview of food webs}

315 Direct observations of feeding links in nature are preferable to inferences based on

316 indirect evidence, experiments, or prior publications from other study $\operatorname{sites}^{25}$. Nevertheless,

317 food web studies are plagued by under-sampling of rare species and links when food webs 
318 are constructed entirely based on direct observation and by over-estimation of links when

319 they are entirely inferred from the literature ${ }^{28,35}$. A yield-effort curve for links as a function of

320 cumulative sampling effort should be reported for all direct observations ${ }^{35}$, but this is still

321 rarely the case in most food web studies ${ }^{28}$. Here, we performed extensive gut content analysis

322 on organisms collected from our study system and used yield-effort curves to assess the

323 completeness of our sampling effort. We supplemented the under-sampled component with

324 inferences from the literature to achieve the optimum balance between under- and over-

325 estimation of true food web structure.

326

327

328

329

330

331

332

333

334

335

336

337

338

339

340

341

\section{Gut content analysis}

We documented 49,324 feeding interactions from 1,128 individual consumers collected from the Hengill streams using gut content analysis. We employed three different approaches: stomach flushing of fish (5,856 interactions from 109 individuals), acid digestion of macroinvertebrates (25,105 interactions from 289 individuals), and dissection of gut contents (18,363 interactions from 730 individuals). Organisms flushed from fish stomachs were immediately stored in $70 \%$ ethanol and later identified under the microscope ${ }^{13,14}$. Immersion of macroinvertebrates in $62 \%$ nitric acid at $65{ }^{\circ} \mathrm{C}$ for 18 hours removes all organic matter except for silicate diatom frustules, enabling accurate identification of diatoms ${ }^{36}$, the major primary producers in the streams ${ }^{13}$. A $1 \mathrm{ml}$ sub-sample of the resulting suspension of diatom frustules was pipetted onto a glass coverslip and allowed to dry before fixing to glass slides by adding a drop of naphrax on a $60{ }^{\circ} \mathrm{C}$ hotplate. We identified the first 100 diatoms (where possible) encountered in a continuous, non-overlapping $100 \mu \mathrm{m}$-wide transect following a fixed route across the slide, which was found to be sufficient for accurately characterising the species present on each $\operatorname{slide}^{36}$. Dissection of gut contents allowed us to quantify predation on other macroinvertebrates and feeding interactions with basal resources 
342 other than diatoms, i.e. coarse particulate organic matter (CPOM, which is $>1 \mathrm{~mm})$, fine

343 particulate organic matter (FPOM, which is $<1 \mathrm{~mm}$ ), macrophytes, filamentous green algae,

344 microscopic green algae, cyanobacteria, and terrestrial subsidies. Invertebrates were dissected 345 at $20 \times$ magnification and the gut contents were mounted onto glass slides with Hoyer's 346 medium. Gut contents were quantified in three randomly chosen fields of view at $200 \times$ 347 magnification on a compound microscope.

\section{Yield-effort curves}

We constructed yield-effort curves using the 'fitspecaccum' function in the 'vegan' package in R 3.5.0, where our community dataset was a matrix with rows as unique consumer guts analysed, columns as resource taxa, and values as the number of times each resource taxon was observed in a consumer's gut. We used 'method = "exact"' and set 'fit' equal to each of the following models: 'arrhenius', 'gleason', 'gitay', 'lomolino', 'asymp', 'gompertz', 'michaelis-menten', 'logis', and 'weibull'. We chose the best fitting model according to AIC and used the 'predict' function in the 'stats' package in R to estimate the predicted number of resource taxa for each consumer, where 'newdata' was the bigger value from twice the number of guts analysed for that consumer and 50 . We carried out this procedure for four different groupings of consumer diet: (1) every consumer species in each stream; (2) every consumer family in each stream; (3) every consumer species in the Hengill region; and (4) every consumer family in the Hengill region.

To construct a food web for a given stream, we started by taking the species list from sampling of that stream in August 2008. We then added links for each species from gut content analysis of those species in that stream. If yield-effort curves suggested that $<95 \%$ of 
365 the diet was described for any species (Fig. S9), we added links for consumers in the same

366 taxonomic family from gut content analysis of those families in that stream. If yield-effort

367 curves suggested that $<95 \%$ of the diet was described for any family (Fig. S10), we added

368 links for each species from gut content analysis of those species across all streams in the

369 Hengill region. If yield-effort curves suggested that $<95 \%$ of the diet was described for any

370 species in the Hengill region (Fig. S11), we added links for consumers in the same taxonomic

371 family from gut content analysis of those families across all streams in the Hengill region. If

372 yield-effort curves suggested that $<95 \%$ of the diet was described for any family in the

373 Hengill region (Fig. S12), we added links described for that species from the literature (Table

374 S6). Just $12.6 \%$ of links were added from the literature, with $43.5 \%$ of links directly observed

375 from the target stream, and the remaining $43.9 \%$ of links directly observed from the Hengill

376 region. From our directly observed links, $74.3 \%$ were specific to each consumer species, with

377 just $25.7 \%$ inferred from the family level. This constitutes one of the most comprehensive

378 food web datasets ever constructed.

Food web properties

Food webs were visualised and properties were calculated using the 'cheddar' package in R. The triangular food webs in Fig. 1a,b and the trivariate food webs in Fig. 2c,d were visualised using the 'PlotWebByLevel' and 'PlotMvN' functions, respectively. Mean trophic level was calculated using the 'ShortWeightedTrophicLevel' function, which is the average of the shortest trophic level of a consumer and $1+$ the mean trophic level of all its trophic resources. This metric has been shown to closely approximate flow-based trophic level, where each link is weighted according to its relative energetic contribution to the consumer's $\operatorname{diet}^{37}$. Connectance was calculated using the 'DirectedConnectance' function, which is the proportion of possible links in a food web that are realised ${ }^{38}$. Mean link length and mean link 
angle were calculated from the 'length' and 'angle' columns under the 'links' data frame returned by the 'NvMTriTrophicStatistics' function. Link lengths describe the distance in mass-abundance space between every consumer and each of its resources in the food web, while link angles describe the biomass of every consumer relative to each of its resources (see Fig. 2a). These metrics are increasingly used to quantify the flux and distribution of

394 biomass through the food $\mathrm{web}^{25,39-42}$ and provide more precise information than biomass pyramids, which only describe the total biomass at each discrete trophic level (see Fig. S6). The ratio of consumer to resource species richness was calculated as the number of consumer species divided by the number of resource species. The difference in the $\log _{10}$ abundanceweighted arithmetic mean body mass of consumers and of resources was taken as the log ratio of consumer to resource body mass. The difference in the $\log _{10}$ mean abundance of consumers and of resources was taken as the log ratio of consumer to resource abundance.

401 The difference in the $\log _{10}$ mean abundance $\times$ body mass of consumers and of resources was taken as the log ratio of consumer to resource biomass. Temperature effects on food web properties were analysed with linear regressions using the ' $I m$ ' function in the 'stats' package in $\mathrm{R}$, with each food web property taken in turn as the dependent variable and stream temperature as the explanatory variable.

\section{Allometric diet breadth model}

The allometric diet breadth model (ADBM) is a model of food web structure based on optimal foraging theory. It predicts the qualitative structure of real food webs, often to a high

409 degree of accuracy ${ }^{16}$. By incorporating the temperature dependence of foraging traits, the

410 model has also been shown as a useful framework for predicting the effects of temperature on

411 food web connectance ${ }^{10}$. The ADBM predicts the diet $k$ of each consumer $j$ that maximises 412 the rate of energy intake: 
$413 \frac{\sum_{i=1}^{k} N_{i} a_{i j} E_{i}}{1+\sum_{i=1}^{k} N_{i} a_{i j} h_{i j}}$,

414 where $N_{i}$ is the density of resource species $i, a_{i j}$ is the attack rate of consumer species $j$ on

415 species $i, \varepsilon_{i}$ is the net energy gained by consumption of an individual of species $i$, and $h_{i j}$ is

416 the time taken for species $j$ to handle an individual of species $i$.

417 The body mass and temperature dependence of $a_{i j}$ can be described as:

$418 \quad a_{0} M_{i}^{a_{i}} M_{j}^{a_{j}} e^{E_{a} \frac{T-T_{0}}{k T T_{0}}}$,

419 where $a_{0}$ is a normalisation constant for attack rate, $M_{i}$ is resource body mass (in $\mathrm{mg}$ ), $M_{j}$ is 420 consumer body mass (in $\mathrm{mg}$ ), $a_{i}$ and $a_{j}$ are allometric exponents, $E_{a}$ is the activation energy of 421 attack rate (in $\mathrm{eV}$ ), $T$ is environmental temperature (in $\mathrm{K}$ ), $T_{0}$ sets the intercept of the 422 temperature relationship at $T_{0}$ rather than at zero Kelvin, and $k$ is the Boltzmann constant $\left(8.618 \times 10^{-5} \mathrm{eV} \mathrm{K}^{-1}\right)$. The value of $E_{i}$ is determined by the proportion of dry-to-wet mass in 424 each organism ${ }^{43,44}, \varepsilon_{i}$, and may vary with temperature ${ }^{45}$, but for simplicity, we assumed here 425 that it would be directly proportional to body mass in all streams ${ }^{10,16}$, i.e. $E_{i}=\varepsilon_{i} M_{i}$. See Table 426 S7 for a list of all parameter values used in the current study and Figs. S13-S16 for an 427 exploration of the sensitivity of key food web properties to the chosen parameter values.

428 The body mass and temperature dependence of $h_{i j}$ can be described as:

429

$\frac{h_{0}}{h_{b}-\frac{M_{i}}{M_{j}}} e^{E_{h} \frac{T-T_{0}}{k T T_{0}}}$,

430 where $h_{0}$ is a normalisation constant for handling time, $h_{b}$ is a critical mass ratio, and $E_{h}$ is the 431 activation energy of handling time (in eV). Note that $h_{i j}=\infty$ if $M_{i} / M_{j} \geq h_{b}$. We let $h_{b}=$ $432 b_{0} M_{j}^{b}$, where $b_{0}=1$ with dimensions that cancel those of $M^{b}$, because resource body mass 
433 has been shown to vary with consumer body mass according to a power-law ${ }^{46}$. Note that we 434 used a ratio handling time function in Equation 3, rather than a power handling time function

435 because the latter is generally shown to have weaker predictive power ${ }^{16}$ and was found to be 436 a poor predictor of empirical food web structure in the Hengill streams. The values for each 437 parameter that were used in the current study are listed in Table S7.

438 It is important to note that the estimates of food web structure based on the ADBM are 439 independent of the empirical quantification of food web structure using dietary analysis. The 440 former relies solely on the body mass and abundance information for each species to 441 determine food web links, whereas the latter determines the links from direct observation in 442 gut contents ( $>87 \%$ of cases) or inference from the literature. Thus, empirical measurements 443 of mean trophic level and connectance are completely independent of the ADBM predictions 444 of these metrics. While mean link length and mean link angle incorporate body mass and 445 abundance information, their values are determined by how consumers and their resources 446 are distributed in mass-abundance space, i.e. there is a major contribution of independent 447 trophic link data to these metrics.

\section{Randomisation scenarios}

We used the ADBM framework to simulate 1,000 food webs for each of our 14 study streams according to three different randomisation scenarios. In the 'sp' scenario, we randomly selected $n$ species from the regional species pool (where $n$ is the number of species in a given stream), with the actual body mass and abundance for each species per stream, or the body mass and abundance from the stream of closest temperature when a species was not

454 found in a stream. This scenario destroyed the ratio of consumer to resource species richness 455 by changing the number of species belonging to each major trophic group (i.e. diatoms, 456 macroinvertebrates, or fish) in each stream, but approximately maintained the ratios of 
consumer to resource body mass and abundance within each stream (Fig. 3a-c). In the ' $M$ ' scenario, we maintained the species found in a stream and their population abundances in that stream, but randomly chose body masses from the same major trophic groups in the regional species pool. This scenario destroyed the ratio of consumer to resource body mass, but approximately maintained the ratios of consumer to resource species richness and abundance within each stream (Fig. 3a-c). In the ' $N$ ' scenario, we maintained the species found in a stream and their mean body masses in that stream, but randomly chose abundances from the same major trophic groups in the regional species pool. This scenario destroyed the ratio of consumer to resource abundance, but approximately maintained the ratios of consumer to resource species richness and body mass within each stream (Fig. 3a-c).

\section{Data and Code Availability:}

The data and $\mathrm{R}$ code that support the findings of this study are available from the first author upon reasonable request.

\section{Additional References:}

35 Cohen, J. E. et al. Improving food webs. Ecology 74, 252-258 (1993).

36 Gordon, T. A. C., Neto-Cerejeira, J., Furey, P. C. \& O’Gorman, E. J. Changes in feeding selectivity of freshwater invertebrates across a natural thermal gradient. Current Zoology 64, 231-242 (2018).

37 Williams, R. J. \& Martinez, N. D. Limits to trophic levels and omnivory in complex food webs: Theory and data. American Naturalist 163, 458-468 (2004).

38 Martinez, N. D. Artifacts or attributes - effects of resolution on the little-rock lake food web. Ecological Monographs 61, 367-392 (1991).

39 Clitherow, L. R., Carrivick, J. L. \& Brown, L. E. Food web structure in a harsh 
glacier-fed river. PloS One 8, e60899 (2013).

$48140 \quad$ Perkins, D. M. et al. Bending the rules: exploitation of allochthonous resources by a top-predator modifies size-abundance scaling in stream food webs. Ecology letters 21, 1771-1780 (2018).

48441 Thompson, M. S. et al. Large woody debris "rewilding" rapidly restores biodiversity 485 in riverine food webs. Journal of applied ecology 55, 895-904 (2018).

48642 Woodward, G. et al. Climate change impacts in multispecies systems: drought alters food web size structure in a field experiment. Philosophical Transactions of the Royal Society B: Biological Sciences 367, 2990-2997 (2012).

48943 James, D. A. et al. A generalized model for estimating the energy density of invertebrates. Freshwater Science 31, 69-77 (2011).

49144 Hartman, K. J. \& Brandt, S. B. Estimating energy density of fish. Transactions of the American Fisheries Society 124, 347-355 (1995).

49345 Converti, A., Casazza, A. A., Ortiz, E. Y., Perego, P. \& Del Borghi, M. Effect of temperature and nitrogen concentration on the growth and lipid content of Nannochloropsis oculata and Chlorella vulgaris for biodiesel production. Chemical Engineering and Processing: Process Intensification 48, 1146-1151 (2009).

49746 Riede, J. O. et al. Stepping in Elton's footprints: a general scaling model for body 498 masses and trophic levels across ecosystems. Ecology Letters 14, 169-178 (2011). 